\title{
TRIPLE FLAMES IN MIXING LAYERS WITH NONUNITY LEWIS NUMBERS
}

\author{
J. DAOU AND A. LIÑÁN \\ Dpto. Motopropulsion y Termofluidodinamica \\ Uni. Politécnica de Madrid \\ E.T.S.I. Aeronáuticos \\ Plaza Cardenal Cisneros, 3 \\ 28040 Madrid, Spain
}

\begin{abstract}
The present paper is devoted to the study of the effects of nonunity Lewis numbers on triple-flame propagation in nonuniform mixtures. For definiteness, the case of a strained reactive mixing layer is considered. The fuel and oxidizer that are fed to the mixing layer are allowed to have different initial temperatures. Specifically, we examine how the triple flames encountered in this context are influenced by (a) the transverse gradients in the temperature and composition of the fresh reactive mixture and (b) by differential-diffusion effects.

The analysis is carried for a single irreversible reaction with a large activation energy and using the thermo-diffusive model. Analytical expressions describing the flame shape, the local burning speed, and the propagation velocity of the triple flame are obtained. In particular, it is found that the Lewis numbers affect the propagation of the triple flame in a way similar to that obtained in the studies of stretched premixed flames. For example, the flame curvature determined by the transverse gradients in the frozen mixing layer leads to flame-front velocities that grow with decreasing values of the Lewis numbers.
\end{abstract}

\section{Introduction}

The presence of different kinds of inhomogeneities in the combustion field is inherent to all combustion applications. An important class of inhomogeneities are the spatial nonuniformities in the composition and temperature of the reactants frequently encountered in unpremixed-combustion devices. Typically, those nonuniformities are essentially transverse to mixing layers, along which flames can propagate, as in lifted jet diffusion flames. Because the combustible mixture varies from lean to rich across a mixing layer, triple flames, consisting of two premixed branches and a trailing diffusion flame, are expected, and therefore, they have been the subject of a number of experimental, analytical, and numerical studies [1-6].

The main purpose of the present investigation is to determine how the propagation of the triple flame is influenced by transverse enthalpy gradients in the fresh mixture and by differential diffusion. We shall select for definiteness the strained mixing layer configuration as a frame for the investigation and adopt additionally the constant density approximation [7] to make the analytical description tractable. The configuration of the study is sketched in Fig. 1, where useful notations are introduced. The velocity field considered is a two-dimensional stagnationpoint type flow with components $v_{y}=-a Y$ and $v_{z}$ $=a \mathrm{Z}$ on the $y$ axis and $z$ axis, respectively, where $a$ is the strain rate.
We shall examine steady flame propagation along the mixing layer in the $x$ direction, described by similarity solutions (i.e., temperature and composition fields) independent of the $z$ coordinate and of time if we use a frame of reference attached to the flame front. In such a frame, the velocity field $\mathbf{v}$ has an

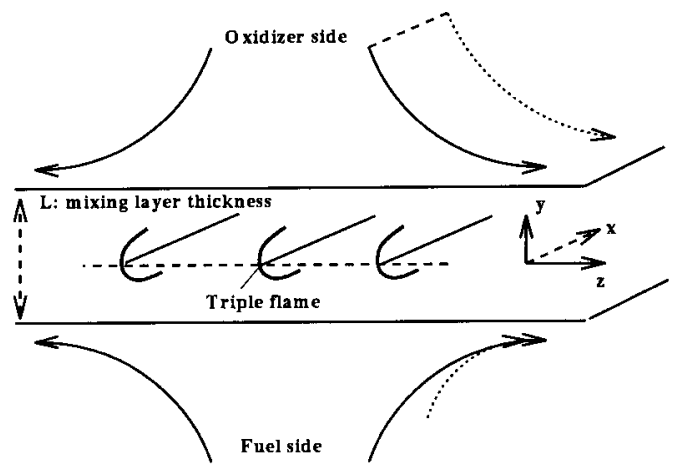

FIG. 1. The strained mixing layer configuration. The fuel stream has temperature $T_{F}$, a fuel mass fraction $y_{F, F}$, and contains no oxidizer. The oxidizer stream has temperature $T_{O}$, an oxidizer mass fraction $y_{O, O}$, and contains no fuel. The density being assumed constant, the velocity field considered is a two-dimensional stagnation-point type flow with components $v_{y}=-a Y$ and $v_{z}=a Z$ on the $y$ axis and $z$ axis, respectively. We shall study herein flame propagation along the mixing layer in the $x$ direction. 
additional $x$ component, $U$, the flame-front velocity that will be an eigenvalue of the problem; hence $\mathbf{v}$ $=(U,-a Y, a Z)$. The triple flames that we thus analyze will correspond, if $U>0$, to ignition fronts that extend diffusion flames to the frozen regions of the mixing layer, or to extinction fronts if $U<0$. Our main concern will be the determination of the flame shape and the front velocity $U$.

\section{Model and General Formulation}

The problem addressed herein is the steady propagation of a flame in a strained mixing layer along the $x$ axis as sketched in Fig. 1. For the sake of simplicity, the following assumptions are adopted. The density $\rho$, the thermal conductivity $\lambda$, the heat capacity $c_{p}$, and the individual species diffusion coefficients are taken as constant. The combustion is represented by a single, irreversible, one-step reaction of the form

$$
\mathrm{F}+s \text { Ox } \rightarrow \mathrm{P}+q
$$

where $\mathrm{F}$ denotes the fuel, Ox the oxidizer, and $\mathrm{P}$ the products. The quantity $s$ denotes the mass of oxidizer consumed and $q$ the heat released, both per unit mass of fuel. The combustion rate, $\omega$, defined as the mass of fuel consumed by unit volume and unit time, is assumed to follow an Arrhenius law of the form

$$
\omega=B \rho^{2} Y_{F} Y_{O} \exp (-E / R T)
$$

where $B, Y_{F}, Y_{O}$, and $E / R$ represent, respectively, the (constant) pre-exponential factor, mass fraction of fuel, mass fraction of oxidizer, and the activation temperature.

For the existence of a steady flame front propagating the reaction in the mixing layer, the activation energy of the reaction must be large enough so that, for strain rates between ignition and extinction values, there are three steady modes of combustion independent of $X$ in the mixing layer, corresponding to the one-dimensional strained diffusion flame. The front joins the upper, strongly burning, and lower, weakly burning modes.

The governing equations are

$$
\begin{gathered}
U \frac{\partial Y_{F}}{\partial X}=D_{F}\left(\frac{\partial^{2} Y_{F}}{\partial X^{2}}+\frac{\partial^{2} Y_{F}}{\partial Y^{2}}\right)-\frac{\omega}{\rho}+a Y \frac{\partial Y_{F}}{\partial Y} \\
U \frac{\partial Y_{O}}{\partial X}=D_{O}\left(\frac{\partial^{2} Y_{O}}{\partial X^{2}}+\frac{\partial^{2} Y_{O}}{\partial Y^{2}}\right)-s \frac{\omega}{\rho}+a Y \frac{\partial Y_{O}}{\partial Y} \\
U \frac{\partial T}{\partial X}=D_{T}\left(\frac{\partial^{2} T}{\partial X^{2}}+\frac{\partial^{2} T}{\partial Y^{2}}\right)+\frac{q}{c_{p}} \frac{\omega}{\rho}+a Y \frac{\partial T}{\partial Y}
\end{gathered}
$$

Here $D_{F}, D_{O}$, and $D_{T}$ denote the diffusion coefficients for the fuel, the oxidizer, and for heat, respectively.
The conditions at $X=-\infty$ correspond to the lower, weakly burning solution independent of $X$, which is very close to the frozen solution if the activation energy is large enough,

$$
\begin{gathered}
Y_{F, f r}=\frac{Y_{F, F}}{2}\left[1-\operatorname{erf}\left(\frac{Y}{\sqrt{2 D_{F} / a}}\right)\right] \\
Y_{O, f r}=\frac{Y_{O, O}}{2}\left[1+\operatorname{erf}\left(\frac{Y}{\sqrt{2 D_{O} / a}}\right)\right] \\
T_{f r}=\frac{T_{F}+T_{O}}{2}+\frac{T_{O}-T_{F}}{2} \operatorname{erf}\left(\frac{Y}{\sqrt{2 D_{T} / a}}\right)
\end{gathered}
$$

where the subscripts $F$ and $O$ refer to the values of the concentrations and temperature on the fuel side and oxidizer side, respectively, which were used in the boundary conditions, at $|Y| \rightarrow \infty, X \rightarrow-\infty$ to obtain (2) and must be used at $|Y| \rightarrow \infty$ for all $X$. The subscript $f r$ is added as a reminder that the values correspond to the fresh (or frozen) side of the mixing layer. Downstream, for $X \rightarrow \infty$, the solution becomes again independent of $X$, corresponding to the onedimensional strong burning solution of the strained diffusion flame.

\section{Large Activation Energy Analysis}

For large activation energies, the flame-front region is expected to be centered around the stoichiometric surface. Upstream, this surface is located at $Y=Y_{s t}$, with $Y_{s t}$ determined by $s Y_{F, f r}\left(Y_{s t}\right)=$ $Y_{O, f r}\left(Y_{s t}\right)$, given by

$$
S \operatorname{erf}\left(\frac{Y_{s t}}{\sqrt{2 D_{F} / a}}\right)+\operatorname{erf}\left(\frac{Y_{s t}}{\sqrt{2 D_{O} / a}}\right)=S-1
$$

where $S \equiv s Y_{F, F} / Y_{O, O}$.

Then, we write the governing equations in terms of the normalized quantities:

$$
y_{F}=\frac{Y_{F}}{Y_{F, s t}}, \quad y_{O}=\frac{Y_{O}}{Y_{O, s t}}, \quad \text { and } \theta=\frac{T-T_{s t}}{T_{a d}-T_{s t}}
$$

Here the subscript $s t$ indicates values at $(X=-\infty$, $Y=Y_{s t}$ ), where the stoichiometric condition $Y_{O, f r}$ $=s Y_{F, f r}$ holds and $T_{a d} \equiv T_{s t}+q Y_{F, s t} / c_{p}$ is the corresponding adiabatic flame temperature.

As reference velocity, we select the laminar burning speed of a stoichiometric planar flame, $S_{L}^{0}$. We have taken $S_{L}^{0}=\left[4 \beta^{-3} Y_{O, s t}\left(\lambda / c_{p}\right) B \exp (-E /\right.$ $\left.\left.R T_{a d}\right)\right]^{1 / 2}$, which is the first approximation for $\beta \gg 1$ of the burning speed of a planar flame corresponding to the stoichiometric conditions prevailing at $(x=$ $-\infty, y=0$ ) and for unity Lewis numbers, as we consider later that the deviation of the Lewis numbers from 1 are $O\left(\beta^{-1}\right)$. We select as length scale $L / \beta$, the expected characteristic value of the radius of curvature of the flame front; here $L=$ 
$\sqrt{2 D_{T} / a}$ is the thickness of the mixing layer and $\beta \equiv$ $E\left(T_{a d}-T_{s}\right) / R T_{a d}^{2}$ the Zeldovich number. A significant variation in the front speed relative to the laminar flame speed, $S_{L}^{0}$, may be expected to take place when $L / \beta$ is of order of the laminar flame thickness $l_{F l}^{0} \equiv \lambda /\left(\rho c_{p} S_{L}^{0}\right)$. We begin by formulating the problem for values of $\varepsilon \equiv l_{F l}^{0} /(L / \beta)$ of order 1. Later, however, we carry out in detail the analysis of the cases, of practical interest, where the radius of curvature is large compared with the laminar flame thickness $l_{F l}^{0}$ (i.e., $\varepsilon \ll 1$ ). In terms of the new nondimensional coordinates $y \equiv \beta\left(Y-Y_{s t}\right) / L$ and $x \equiv \beta X / L$, the nondimensional governing equations are

$$
\begin{aligned}
U \frac{\partial y_{F}}{\partial x}=\frac{\varepsilon}{\mathrm{Le}_{\mathrm{F}}}\left(\frac{\partial^{2} y_{F}}{\partial x^{2}}+\frac{\partial^{2} y_{F}}{\partial y^{2}}\right) & -\varepsilon^{-1} \tilde{\omega} \\
& +\frac{2 \varepsilon}{\beta}\left(\eta_{s}+\frac{y}{\beta}\right) \frac{\partial y_{F}}{\partial y} \\
U \frac{\partial y_{O}}{\partial x}=\frac{\varepsilon}{\mathrm{Le}_{\mathrm{O}}}\left(\frac{\partial^{2} y_{O}}{\partial x^{2}}+\frac{\partial^{2} y_{O}}{\partial y^{2}}\right)-\varepsilon^{-1} \tilde{\omega} & +\frac{2 \varepsilon}{\beta}\left(\eta_{s}+\frac{y}{\beta}\right) \frac{\partial y_{O}}{\partial y} \\
U \frac{\partial \theta}{\partial x}=\varepsilon\left(\frac{\partial^{2} \theta}{\partial x^{2}}+\frac{\partial^{2} \theta}{\partial y^{2}}\right)+\varepsilon^{-1} \tilde{\omega} & +\frac{2 \varepsilon}{\beta}\left(\eta_{s}+\frac{y}{\beta}\right) \frac{\partial \theta}{\partial y}
\end{aligned}
$$

to be solved with the upstream boundary conditions 2 , which when written in terms of $y$ would simplify to

$$
y_{F}=1-\frac{\gamma_{F}}{\beta} y, y_{O}=1+\frac{\gamma_{O}}{\beta} y, \theta=\frac{\gamma_{\theta}}{\beta} y
$$

if we could confine our attention to the flame-front region, $y \sim 1$, and neglect terms of order $\beta^{-2}$. Here $\gamma_{F}, \gamma_{O}$, and $\gamma_{\theta}$ are given by

$$
\begin{gathered}
\gamma_{F}=\frac{2 \sqrt{L e_{F}} \exp \left(-\eta_{s}^{2} L e_{F}\right)}{\sqrt{\pi}\left[1-\operatorname{erf}\left(\eta_{s} \sqrt{L e_{F}}\right)\right]} \\
\gamma_{O}=\frac{2 \sqrt{L e_{O}} \exp \left(-\eta_{s}^{2} L e_{O}\right)}{\sqrt{\pi}\left[1+\operatorname{erf}\left(\eta_{s} \sqrt{L e_{O}}\right)\right]} \\
\gamma_{\theta}=\frac{2 \exp \left(-\eta_{s}^{2}\right)}{\sqrt{\pi}\left[1-\operatorname{erf}\left(\eta_{s} \sqrt{L e_{F}}\right)\right]} \Psi
\end{gathered}
$$

$L e_{F} \equiv D_{T} / D_{F}$ and $L e_{O} \equiv D_{T} / D_{O}$ are the Lewis numbers of the fuel and of the oxidizer, respectively, and $\eta_{s}$ and $Y$ are two nondimensional parameters characterizing the location of the stoichiometric surface and the transverse temperature gradient in the frozen mixture:

$$
\eta_{s} \equiv \frac{Y_{s t}}{\sqrt{2 D_{T} / a}} \quad \text { and } Y \equiv \frac{T_{O}-T_{F}}{q Y_{F, F} / c_{p}}
$$

The nondimensional reaction rate $\tilde{\omega}$ is given by

$$
\tilde{\omega}=\frac{\beta^{3}}{4} y_{F} y_{O} \exp \left[\frac{-\beta(1-\theta)}{1+\alpha_{h}(\theta-1)}\right]
$$

where $\alpha_{h} \equiv\left(T_{a d}-T_{s t}\right) / T_{a d}$ is a heat-release parameter. It is clear that one of the three parameters in equations 6 , say $\gamma_{F}$, can be of course eliminated, by redefining the length scale (replacing $L$ by $\gamma_{F} L$, for example)

In the limit $\beta \rightarrow \infty$, the reaction is confined to an infinitely thin reaction sheet, which we refer to as the flame surface and which is given by $F(x, y)=x$ $-f(y)=0$. The analysis is restricted to the nearequidiffusion cases for which $l_{F} \equiv \beta\left(L e_{F}-1\right)$ and $l_{O} \equiv \beta\left(L e_{O}-1\right)$ are of order 1 . Then (see Ref. [8], for example) appropriate jump conditions can be derived across the reaction sheet, and the problem can be reformulated in a way free from the presence of $\beta$. We use a coordinate system attached to the flame,

$$
\xi=x-f(y), \quad y=y
$$

so that the flame surface is located at $\xi=0$; in terms of the new coordinates, the Laplacian $\Delta=\partial^{2} / \partial x^{2}+$ $\partial^{2} / \partial y^{2}$ takes the form

$$
\begin{aligned}
\Delta=\left(1+f^{\prime 2}\right) & \frac{\partial^{2}}{\partial \xi^{2}} \\
& +\frac{\partial^{2}}{\partial y^{2}}-f^{\prime \prime} \frac{\partial}{\partial \xi}-2 f^{\prime} \frac{\partial}{\partial \xi \partial y}
\end{aligned}
$$

The dependent variables are expanded in terms of $\beta^{-1}$ as

$$
\begin{aligned}
y_{F} & =y_{F}^{0}+\beta^{-1} y_{F}^{1}+\ldots, \quad y_{O}=y_{O}^{0} \\
& +\beta^{-1} y_{O}^{1}+\ldots, \quad \theta=\theta^{0}+\beta^{-1} \theta^{1}+\ldots
\end{aligned}
$$

Note that superscripts are used to indicate the different orders of the expansions in terms of $\beta^{-1}$. Expansions in terms of $\varepsilon$ introduced later are expressed by subscripts to avoid confusion. For $\beta \gg 1$, the reaction zone is thin, of extent $\xi \sim \varepsilon / \beta$, so that it can be described by the diffusive-reactive balance obtained by dropping the convective terms in equation 4 and retaining only the first term on the right-hand side (RHS) of equation 10.

Because the boundary conditions (5) introduce $O\left(\beta^{-1}\right)$ nonuniformities in the concentration of the reactants and their temperature, the corresponding variations in the burned gas relative to the uniform case (that is, the planar flame case) are also expected to be $O\left(\beta^{-1}\right)$. Thus, we write in the reaction zone and behind it in the burned gas, that is, for $\xi \geq 0$,

$$
\theta^{0}=1, \quad y_{F}^{0}=0, \quad y_{O}^{0}=0
$$

Defining now $Z_{F}$ and $Z_{O}$ by

$$
Z_{F} \equiv \theta+y_{F}, \quad Z_{O} \equiv \theta+y_{O}
$$

it follows from (4) and (9) that 


$$
\begin{aligned}
U \frac{\partial Z_{F}}{\partial \xi} & =\varepsilon \Delta Z_{F}-\varepsilon \frac{l_{F}}{\beta} \Delta y_{F} \\
& +\frac{2 \varepsilon}{\beta}\left(\eta_{s}+\frac{y}{\beta}\right)\left[\frac{\partial Z_{F}}{\partial y}-f^{\prime}(y) \frac{\partial Z_{F}}{\partial \xi}\right] \\
U \frac{\partial Z_{O}}{\partial \xi} & =\varepsilon \Delta Z_{O}-\varepsilon \frac{l_{O}}{\beta} \Delta y_{O} \\
+ & \frac{2 \varepsilon}{\beta}\left(\eta_{s}+\frac{y}{\beta}\right)\left[\frac{\partial Z_{O}}{\partial y}-f^{\prime}(y) \frac{\partial Z_{O}}{\partial \xi}\right]
\end{aligned}
$$

The expansions of $Z_{F}$ and $Z_{O}$ in terms of $\beta^{-1}$ are of the form

$$
\begin{aligned}
Z_{F}=1+\beta^{-1} h(\xi, y) & +\ldots, Z_{O}=1 \\
+ & \beta^{-1} k(\xi, y)+\ldots
\end{aligned}
$$

because the upstream nonuniformities in $y_{F}, y_{O}$, and $\theta$ are of order $\beta^{-1}$, so that

$$
\begin{array}{ll}
\theta^{0}+y_{F}^{0}=1, & \theta^{0}+y_{O}^{0}=1 \\
h \equiv \theta^{1}+y_{F}^{1}, & k \equiv \theta^{1}+y_{O}^{1}
\end{array}
$$

where the last line in (16) defines the excess enthalpy functions $h$ and $k$ appearing in (15). Behind the reaction sheet $\xi>0$, reaction ceases because one of the reactants has been depleted. Thus, we can write for $\xi>0$ :

$$
\begin{gathered}
\theta^{1}=h, \quad y_{O}^{1}=k-h \text { (fuel-lean side) } \\
\theta^{1}=k, \quad y_{F}^{1}=h-k \text { (fuel-rich side) }
\end{gathered}
$$

Now, on using (4), (14), and (15), we obtain the governing equations for $\theta^{0}, h$, and $k$, valid at both sides of the reaction sheet*:

$$
\begin{gathered}
U \frac{\partial \theta^{0}}{\partial \xi}=\varepsilon \Delta \theta^{0} \\
U \frac{\partial h}{\partial \xi}=\varepsilon \Delta h+\varepsilon l_{F} \Delta \theta^{0} \\
U \frac{\partial k}{\partial \xi}=\varepsilon \Delta k+\varepsilon l_{O} \Delta \theta^{0}
\end{gathered}
$$

Note that the terms associated with strain (the last terms in equations 4 and 14) have dropped out in the preceding equations: those are of order $\beta^{-2}$, which follows from (16a). The jump conditions resulting from integration of the quasi-planar reactiondiffusion equations are

$$
\begin{gathered}
{\left[\theta^{0}\right]=[h]=[k]=0} \\
{\left[\frac{\partial h}{\partial \xi}\right]=-l_{F}\left[\frac{\partial \theta^{0}}{\partial \xi}\right]}
\end{gathered}
$$

\footnotetext{
*The last two equations in (18) are in fact also valid through the reaction sheet
}

$$
\begin{gathered}
{\left[\frac{\partial k}{\partial \xi}\right]=-l_{O}\left[\frac{\partial \theta^{0}}{\partial \xi}\right]} \\
\varepsilon \sqrt{1+f^{\prime 2}}\left[\frac{\partial \theta^{0}}{\partial \xi}\right]=-\left[1+\frac{1}{2}(\mu-\sigma)\right]^{1 / 2} \exp (\sigma / 2)
\end{gathered}
$$

to be satisfied at $\xi=0$. Here we use the notations

$$
\begin{aligned}
\sigma & \equiv \theta_{b}^{1}=h\left(0^{+}, y\right), \\
\mu & =k\left(0^{+}, y\right) \text { (fuel-lean side) } \\
\sigma & \equiv \theta_{b}^{1}=k\left(0^{+}, y\right), \\
\mu & =h\left(0^{+}, y\right) \text { (fuel-rich side) }
\end{aligned}
$$

The bracket applied to any quantity $\psi$ denotes the difference between the values of $\psi$ at both sides of the reaction sheet, namely, $[\psi] \equiv \psi\left(\xi=0^{+}, y\right)-$ $\psi\left(\xi=0^{-}, y\right)$. Also, the boundary conditions at $\xi=$ $-\infty$ for $\theta^{0}, h$, and $k$ follow from (5), (11), and the definition of $h$ and $k$ :

$$
\begin{aligned}
\theta^{0} & =0, \quad h=\left(-\gamma_{F}+\gamma_{\theta}\right) y, \\
k & =\left(\gamma_{O}+\gamma_{\theta}\right) y \text { as } \xi \rightarrow-\infty
\end{aligned}
$$

Note that because we consider the deviation of the Lewis numbers from 1 to be $O\left(\beta^{-1}\right)$, the coefficients $\gamma_{F}, \gamma_{O}$, and $\gamma_{\theta}$ in (6) must be slightly simplified to become

$$
\begin{aligned}
& \gamma_{F}=\frac{2 \exp \left(-\eta_{s}^{2}\right)}{\sqrt{\pi}\left[1-\operatorname{erf}\left(\eta_{s}\right)\right]}, \\
& \gamma_{O}=\frac{2 \exp \left(-\eta_{s}^{2}\right)}{\sqrt{\pi}\left[1+\operatorname{erf}\left(\eta_{s}\right)\right]}, \\
& \gamma_{\theta}=\frac{2 \exp \left(-\eta_{s}^{2}\right)}{\sqrt{\pi}\left[1-\operatorname{erf}\left(\eta_{s}\right)\right]} Y
\end{aligned}
$$

The boundary conditions at $|y| \rightarrow \infty$ and $\xi$ finite are also given by equations 21 . These are exact solutions of equations 18 and 19, if we restrict our analysis to the only cases that we consider, where $\gamma_{\theta}-\gamma_{F}<0$ and $\gamma_{\theta}+\gamma_{O}>0$.

In summary, the limit $\beta \rightarrow \infty$ and the assumption that the Lewis numbers are close to unity allow us to replace the original conservation equations and associated boundary and jump conditions to be verified by $\theta, y_{F}$, and $y_{O}$ by similar expressions written in terms of $\theta^{0}, h$, and $k$. Our task now is to solve equations 18 , valid at both sides of the reaction sheet $(\xi<0$ and $\xi>0)$, subject to the jump conditions (19) at $\xi=0$ and the boundary conditions (21) at $\xi$ $=-\infty$ and at $|y| \rightarrow \infty$. Downstream, at $\xi \rightarrow \infty$, we simply require the derivatives with respect to $\xi$ to decay to zero.

The solution of the foregoing problem will provide the flame-front velocity $U$ is terms of $\varepsilon \gamma_{F}, \gamma_{O} / \gamma_{F}, \gamma_{\theta} /$ $\gamma_{F}, l_{F}$, and $l_{O}$. We do not include in this paper the 
results of the numerical analysis of the cases where $\varepsilon \sim 1$. We do, however, describe with some detail the limiting case where $\varepsilon \ll 1$, corresponding to large values of the Damköhler number, which is important in applications.

Before carrying out this analysis in the following sections, we mention a further simplification of the equations, which will nevertheless not be used in the present paper. It can be shown, indeed, that the functions $h$ and $k$ can be obtained in terms of a single function $g(\xi, y)$ by the relations

$$
\begin{aligned}
& h=\left(\gamma_{\theta}-\gamma_{F}\right) y+l_{F}\left(g-\theta^{0}\right), \\
& k=\left(\gamma_{\theta}+\gamma_{O}\right) y+l_{O}\left(g-\theta^{0}\right)
\end{aligned}
$$

where $g$ is continuous and has continuous gradients at the reaction sheet and is governed by

$$
U \frac{\partial g}{\partial \xi}=\varepsilon \Delta g+U \frac{\partial \theta^{0}}{\partial \xi}
$$

satisfying the boundary conditions $g \rightarrow 0$ both at $\xi$ $\rightarrow-\infty$ and $|y| \rightarrow \infty$ and bounded at $\xi \rightarrow \infty$. The perturbation, $\sigma$, in the premixed flame temperature can be obtained from the relations (23) and the value, $g_{P}$, of $g$ at $\xi=0$. Behind the lean and rich branches of the premixed flame front, there is also a thin diffusion-controlled reaction layer or diffusion flame, $g=g_{D}$, where $g_{D}$ is given by equations 23 with $h=k$ and $\theta^{0}=1$.

\section{The Limit Cases $\varepsilon \ll 1$}

In the limit $\varepsilon \rightarrow 0$, the flame, including its preheat zone, can be viewed as a surface of discontinuity located at $\xi=0$. For $\varepsilon \ll 1$, the preheat zone upstream of the reaction sheet is a thin layer of thickness of order $\varepsilon$. Outside this layer, diffusion and heat conduction can be neglected in first approximation. For small values of $\varepsilon$, we describe the solution in the form of expansions in $\varepsilon$ of the form $f=f_{0}+\varepsilon f_{1}, U$ $=U_{0}+\varepsilon U_{1}$, and similar expansions for $\theta, h, k$ written for the different regions.

\section{Outer Solutions on Both Sides of the Flame}

For $\xi<0$ and $\xi>0$, we seek outer expansions in the form

$$
\begin{aligned}
\theta^{0} & =\Theta_{0}+\varepsilon \Theta_{1}+\ldots, \quad h=H_{0} \\
& +\varepsilon H_{1}+\ldots, \quad k=K_{0}+\varepsilon K_{1}+\ldots
\end{aligned}
$$

which we substitute into equations 18 . We then obtain in first approximation $U_{0} \partial \Theta_{0} / \partial \xi=U_{0} \partial H_{0} / \partial \xi=$ $U_{0} \partial K_{0} / \partial \xi=0$. Thus $\Theta_{0}, H_{0}$, and $K_{0}$ are independent of $\xi$ on both sides of the flame, although they have jumps across the flame. More precisely, when conditions 21 and 12 are used, one finds

$$
\theta^{0}=\theta_{0}=\left\{\begin{array}{lll}
0 & \text { for } & \xi<0 \\
1 & \text { for } & \xi>0
\end{array}\right.
$$

and

$$
\begin{aligned}
h=H_{0} & =\left(-\gamma_{F}+\gamma_{\theta}\right) y \text { and } k \\
& =K_{0}=\left(\gamma_{O}+\gamma_{\theta}\right) y, \text { for } \xi<0
\end{aligned}
$$

\section{The Structure of the Flame}

We analyze now the thin region around $\xi=0$ where diffusive effects should be retained. To study this zone, we use the stretching transformation $\xi=$ $\varepsilon \zeta$. We seek expansions in the form

$$
\begin{array}{r}
\theta^{0}=\theta_{0}+\varepsilon \theta_{1}+\ldots, \quad h=h_{0}+\varepsilon h_{1} \\
+\ldots, \quad k=k_{0}+\varepsilon k_{1}+\ldots
\end{array}
$$

When using these expansions with the jump conditions at the reaction sheet, we obtain

$$
\begin{gathered}
{\left[\theta_{0}\right]=\left[h_{0}\right]=\left[k_{0}\right]=0} \\
{\left[\frac{\partial h_{0}}{\partial \zeta}\right]=-l_{F}\left[\frac{\partial \theta_{0}}{\partial \zeta}\right]} \\
{\left[\frac{\partial k_{0}}{\partial \zeta}\right]=-l_{O}\left[\frac{\partial \theta_{0}}{\partial \zeta}\right]} \\
\left.\sqrt{1+f_{0}^{\prime 2}}\left[\frac{\partial \theta_{0}}{\partial \zeta}\right]=-\left[1+\frac{1}{2}\left(\mu_{0}-\sigma_{0}\right)\right]\right]^{1 / 2} \exp \left(\sigma_{0} / 2\right)
\end{gathered}
$$

and

$$
\begin{gathered}
{\left[\theta_{1}\right]=\left[h_{1}\right]=\left[k_{1}\right]=0} \\
{\left[\frac{\partial h_{1}}{\partial \zeta}\right]=-l_{F}\left[\frac{\partial \theta_{1}}{\partial \zeta}\right]} \\
{\left[\frac{\partial k_{1}}{\partial \zeta}\right]=-l_{O}\left[\frac{\partial \theta_{1}}{\partial \zeta}\right]} \\
\left.\frac{\partial \theta_{1}}{\partial \zeta}\right]=(A-F)\left[\frac{\partial \theta_{0}}{\partial \zeta}\right]
\end{gathered}
$$

where $A$ and $F$ are defined by

$$
\begin{aligned}
A & \equiv \frac{\sigma_{1}}{2}+\frac{\left(\mu_{1}-\sigma_{1}\right) / 4}{1+\left(\mu_{0}-\sigma\right) / 2}, \\
F & \equiv \frac{f_{0}^{\prime} f_{1}^{\prime}}{1+f_{0}^{\prime 2}}
\end{aligned}
$$

In equations 29 and 30 , we have introduced the expansions $\left(\sigma=\sigma_{0}+\varepsilon \sigma_{1}+\ldots\right)$ and $\left(\mu=\mu_{0}+\varepsilon \mu_{1}\right.$ $+\ldots)$, which on using equation 20 are given by

$$
\begin{array}{r}
\sigma_{i}=h_{i}\left(0^{+}, y\right), \quad \mu_{i}=k_{i}\left(0^{+}, y\right) \\
\text { (fuel-lean side) }(i=0,1, \ldots) \\
\sigma_{i}=k_{i}\left(0^{+}, y\right), \quad \mu_{i}=h_{i}=h_{i}\left(0^{+}, y\right)
\end{array}
$$

(fuel-rich side) 
Using equation 28 and the conservation equations 18 written in terms of $y$ and the inner variable $\zeta=$ $\xi / \varepsilon$ yields to leading order

$$
\begin{gathered}
U_{0} \frac{\partial \theta_{0}}{\partial \zeta}-\left(1+f_{0}^{\prime 2}\right) \frac{\partial^{2} \theta_{0}}{\partial \zeta^{2}}=0 \\
U_{0} \frac{\partial h_{0}}{\partial \zeta}-\left(1+f_{0}^{\prime 2}\right) \frac{\partial^{2} h_{0}}{\partial \zeta^{2}}=l_{F}\left(1+f_{0}^{\prime 2}\right) \frac{\partial^{2} \theta_{0}}{\partial \zeta^{2}} \\
U_{0} \frac{\partial k_{0}}{\partial \zeta}-\left(1+f_{0}^{\prime 2}\right) \frac{\partial^{2} k_{0}}{\partial \zeta^{2}}=l_{O}\left(1+f_{0}^{\prime 2}\right) \frac{\partial^{2} \theta_{0}}{\partial \zeta^{2}}
\end{gathered}
$$

The general solution of (33a) is $\theta_{0}=A+B \exp$ $(\alpha \zeta)$, where

$$
\alpha \equiv \frac{U_{0}}{1+f_{0}^{\prime 2}}
$$

and $A$ and $B$ are constants to be determined at both sides of the reaction sheet. Thus, for $\zeta>0$, we must have $\theta_{0}=1$, which is the bounded solution that matches the anticipated outer solution in the burned gas (26). For $\zeta<0$, the solution must be $\theta_{0}=\exp$ $(\alpha \zeta)$ in order to satisfy the matching requirement with the upstream outer solution $(26 \mathrm{a})$ and the continuity requirement $\left[\theta_{0}\right]=0$ at $\zeta=0$. Thus, we have

$$
\theta_{0}=\left\{\begin{array}{cc}
\exp (\alpha \zeta) & \text { for } \zeta<0 \\
1 & \text { for } \zeta>0
\end{array}\right.
$$

Similarly $h_{0}$ and $k_{0}$ will be independent of $\zeta$ so as to be bounded and given by

$$
\begin{gathered}
h_{0}=\left(-\gamma_{F}+\gamma_{\theta}\right) y-\alpha l_{F} \zeta \exp (\alpha \zeta) \quad \zeta \leq 0 \\
k_{0}=\left(\gamma_{O}+\gamma_{\theta}\right) y-\alpha l_{O} \zeta \exp (\alpha \zeta) \quad \zeta \leq 0
\end{gathered}
$$

if we use the matching conditions with the upstream solution (27) and the jump conditions (29b) and (29c). Because $h_{0}$ and $k_{0}$ are continuous at $\zeta=0$, it also follows that $h_{0}=\left(-\gamma_{F}+\gamma_{\theta}\right) y$ and $k_{0}=\left(\gamma_{O}\right.$ $\left.+\gamma_{\theta}\right) y$ in the burned gas, $\zeta>0$. The jump condition (29d) allows us to determine the local burning speed* $S_{0} \equiv U_{0} /\left(1+f_{0}^{\prime 2}\right)^{1 / 2}$, a quantity that appears as the left-hand side (LHS) of (29d) after using (34) and (35). The RHS of (29d) is known, because $\sigma_{0}$ and $\mu_{0}$ are available from (32) and (36), namely,

$$
\begin{aligned}
\sigma_{0}= & \left(-\gamma_{F}+\gamma_{\theta}\right) y, \quad \mu_{0}=\left(\gamma_{O}+\gamma_{\theta}\right) y \\
& (\text { fuel-lean side }) \\
\sigma_{0}= & \left(\gamma_{O}+\gamma_{\theta}\right) y, \quad \mu_{0}=\left(-\gamma_{F}+\gamma_{\theta}\right) y
\end{aligned}
$$

(fuel-rich side)

Consequently, the local burning speed is given by

*The local burning speed $S$ is defined as the component of the fluid velocity ahead of the flame normal to the flame surface, $S=\left[U \mathbf{i}-2(\varepsilon / \beta)\left(\eta_{s}+y / \beta\right) \mathbf{j}\right] \mathbf{n}=U /\left[1+f^{\prime}(y)^{2}\right]^{1 / 2}$ $+O\left(\beta^{-1}\right)$, where $\mathbf{n}=\left[\mathbf{i}-f^{\prime}(y) \mathbf{j}\right] /\left[1+f^{\prime}(y)\right]^{1 / 2}$ is the unit vector normal to the flame pointing to the burned gas.

$$
\begin{aligned}
& S_{0}=\frac{U_{0}}{\sqrt{1+f_{0}^{\prime}}}= \\
& \left\{\begin{array}{cl}
e^{-\gamma \Gamma y}(1+\gamma y)^{1 / 2} & \text { for } y>0 \text { (fuel-lean) } \\
e^{\gamma(1-\Gamma) y}(1-\gamma y)^{1 / 2} & \text { for } y<0 \text { (fuel-rich) }
\end{array}\right.
\end{aligned}
$$

where the new parameters $\Gamma$ and $\gamma$ are defined by

$$
\Gamma \equiv \frac{\gamma_{F}-\gamma_{\theta}}{\gamma_{F}+\gamma_{O}}, \quad \gamma \equiv \frac{\gamma_{F}+\gamma_{O}}{2}
$$

and can be expressed in terms of $\eta_{s}$ and $Y$ given in (7) as

$$
\begin{aligned}
\gamma=\frac{2 \exp \left(-\eta_{s}^{2}\right)}{\sqrt{\pi}\left(1-\operatorname{erf}\left(\eta_{s}\right)^{2}\right)} \text { and } \Gamma \\
=\frac{1+\operatorname{erf}\left(\eta_{s}\right)}{2}(1-\Gamma)
\end{aligned}
$$

Equation 37, involving the eigenvalue $U_{0}$, determines the flame shape, $f_{0}(y)$, in first approximation. For fixed values of $\Gamma$ and $\gamma,(37)$ admits solutions for infinitely many values of $U_{0}$, but only for a single value of $U_{0}$, the solution will not present a cusp (i.e., a discontinuous slope) at its leading edge, as may be expected to be the case for freely propagating triple flames. This fixes the value of $U_{0}$, requiring that the derivative $\mathrm{f}_{0}^{\prime}$ is zero at the leading edge, a criterion which has been used by Dold [2]. We thus find that the velocity of propagation of the triple flame only depends on $\Gamma$ and is given by

$$
U_{0}= \begin{cases}e^{(\Gamma-1 / 2) /} \sqrt{2 \Gamma} & \text { for } 0<\Gamma \leq 1 / 2 \\ e^{(-\Gamma+1 / 2)} / \sqrt{2(1-\Gamma)} & \text { for } 1 / 2 \leq \Gamma<1\end{cases}
$$

This is also the maximum burning speed, obtained at the leading edge located (in first approximation) at

$$
y^{*}= \begin{cases}(1-2 \Gamma) / 2 \gamma \Gamma & 0<\Gamma \leq 1 / 2 \\ (1-2 \Gamma) / 2 \gamma(1-\Gamma) & 1 / 2 \leq \Gamma<1\end{cases}
$$

It is easy to check that a leading edge exists only for values of $\Gamma$ strictly between 0 and 1 . In view of (39), the condition $0<\Gamma<1$ is always fulfilled when $\mathrm{Y}$ $=0$, that is, whenever the fuel side and the oxidizer side have equal temperatures. In the general case, the condition $0<\Gamma<1$, ensuring the existence of a leading edge, can be written in terms of the parameter $Y$ and can be shown to be equivalent to the statement that a leading edge exists only if the temperature at the oxidizer side and that at the fuel side are less than the Burke-Schumann temperature of the diffusion flame in the mixing layer. It is important to point out that our conclusion about the existence of a leading edge is based on a linearization about the stoichiometric line and on the existence of a peak in $S_{0}(y)$. We do not discuss here the nongeneric case-in which the temperature of one of the 
feed streams is hotter than the Burke-Schumann temperature-for which $S_{0}(y)$ has no maximum.

\section{Second Approximation for the Solution}

We have just determined the first approximation to the local burning speed, the triple-flame propagation velocity, and, by using (37), the flame shape. Our aim now is to obtain the next approximation, that is, the following terms in the expansions of these quantities in terms of $\varepsilon$, and thus the influence of Lewis numbers on the propagation of the triple flame. Because of space limitations, the details of the analysis, which is straightforward following the lines of the previous section, will be kept more concise.

From (18), we obtain

$$
\begin{gathered}
U_{0} \frac{\partial \theta_{1}}{\partial \zeta}-\left(1+f_{0}^{\prime 2}\right) \frac{\partial^{2} \theta_{1}}{\partial \zeta^{2}} \\
=L\left(\theta_{0}\right)-U_{1} \frac{\partial \theta_{0}}{\partial \zeta} \\
U_{0} \frac{\partial h_{1}}{\partial \zeta}-\left(1+f_{0}^{\prime 2}\right) \frac{\partial^{2} h_{1}}{\partial \zeta^{2}}=L\left(h_{0}+l_{F} \theta_{0}\right) \\
-U_{1} \frac{\partial h_{0}}{\partial \zeta}+l_{F}\left(1+f_{0}^{\prime 2}\right) \frac{\partial^{2} \theta_{1}}{\partial \zeta^{2}}, \\
U_{0} \frac{\partial k_{1}}{\partial \zeta}-\left(1+f_{0}^{\prime 2}\right) \frac{\partial^{2} k_{1}}{\partial \zeta^{2}}=L\left(k_{0}+l_{O} \theta_{0}\right) \\
-U_{1} \frac{\partial k_{0}}{\partial \zeta}+l_{O}\left(1+f_{0}^{\prime 2}\right) \frac{\partial^{2} \theta_{1}}{\partial \zeta^{2}}
\end{gathered}
$$

where $L$ is given by

$$
L \equiv 2 f_{0}^{\prime} f_{1}^{\prime} \frac{\partial^{2}}{\partial \zeta^{2}}-f_{0}^{\prime \prime} \frac{\partial}{\partial \zeta}-2 f_{0}^{\prime} \frac{\partial^{2}}{\partial y \partial \zeta}
$$

Downstream of the reaction sheet, it is found that

$$
\begin{aligned}
\theta_{1}=0, \quad h_{1}=h_{1}^{+} \equiv & h_{1}(0, y) \quad k_{1}=k_{1}^{+} \\
& \equiv k_{1}(0, y) \quad(\zeta \geq 0)
\end{aligned}
$$

where the perturbations in the excess enthalpies, $h_{1}^{+}$and $k_{1}^{+}$, are as yet undetermined.

Integration of (42) from $\zeta=-\infty$ to $\zeta=0^{-}$yields, on using (26), (27), and (30a),

$$
\begin{gathered}
\left(1+f_{0}^{\prime 2}\right)\left[\frac{\partial \theta_{1}}{\partial \zeta}\right]=I_{\theta}-U_{1} \\
U_{0} h_{1}^{+}+\left(1+f_{0}^{\prime 2}\right)\left[\frac{\partial h_{1}}{\partial \zeta}\right] \\
=-l_{F}\left(1+f_{0}^{\prime 2}\right)\left[\frac{\partial \theta_{1}}{\partial \zeta}\right]+I_{h}+l_{F} I_{\theta}
\end{gathered}
$$

$$
\begin{aligned}
& U_{0} k_{1}^{+}+\left(1+f_{0}^{\prime 2}\right)\left[\frac{\partial k_{1}}{\partial \zeta}\right] \\
& =-l_{O}\left(1+f_{0}^{\prime 2}\right)\left[\frac{\partial \theta_{1}}{\partial \zeta}\right]+I_{k}+l_{O} I_{\theta}
\end{aligned}
$$

where we have introduced the notations

$$
\begin{array}{r}
I_{\theta}=\int_{-\infty}^{0} L\left(\theta_{0}\right) d \zeta \quad I_{h}=\int_{-\infty}^{0} L\left(h_{0}\right) d \zeta \quad I_{k} \\
=\int_{-\infty}^{0} L\left(k_{0}\right) d \zeta
\end{array}
$$

From (35) and (36), the integrals in (45) are found to be given by

$$
\begin{aligned}
& I_{\theta}=2 U_{0} F-f_{0}^{\prime \prime}, \quad I_{h}=-2 l_{F} U_{0} F, \\
& I_{k}=-2 l_{O} U_{0} F
\end{aligned}
$$

Then, with the aid of (30) and use of (35), equations 44 reduce to

$$
\begin{aligned}
U_{0} h_{1}^{+} & =-l_{F} f_{0}^{\prime \prime} \\
U_{0} k_{1}^{+} & =-l_{O} f_{0}^{\prime \prime} \\
U_{0} F-U_{1} & =f_{0}^{\prime \prime}-U_{0} A
\end{aligned}
$$

involving $h_{1}^{+}, k_{1}^{+}$, the perturbation $U_{1}$ in front velocity, and the functions $\mathrm{A}$ and $\mathrm{F}$ of $y$ defined in (31), namely,

$$
A \equiv \frac{\sigma_{1}}{2}+\frac{\left(\mu_{1}-\sigma_{1}\right) / 4}{1+\left(\mu_{0}-\sigma_{0}\right) / 2}, \quad F \equiv \frac{f_{0}^{\prime} f_{1}^{\prime}}{1+f_{0}^{\prime 2}}
$$

At this stage, we have three equations for four unknowns. However, it is possible to determine directly the perturbation in flame velocity, $U_{1}$, if we use the system of equations (46) at the leading edge of the flame, $y^{*}$, where $F=0$ because $f_{0}^{\prime}\left(y^{*}\right)=0$.

Thus, we obtain

$$
\begin{aligned}
U_{1}= & -\left[1+\frac{l_{F}}{2}-\frac{\left(l_{F}-l_{O}\right) / 4}{1+\gamma y^{*}}\right] \sqrt{2} \gamma \Gamma \\
& (0<\Gamma \leq 1 / 2) \\
= & -\left[1+\frac{l_{O}}{2}-\frac{\left(l_{O}-l_{F}\right) / 4}{1+\gamma y^{*}}\right] \sqrt{2} \gamma(1-\Gamma) \\
& (1 / 2 \leq \gamma<1)
\end{aligned}
$$

With $U_{1}$ thus determined, we can use again the system of equations (46) for values of $y$ different from $y^{*}$ to calculate $h_{1}^{+}, k_{1}^{+}$, and $f_{1}^{\prime}$. The results can be used in particular to calculate the temperature along the flame front, $\theta_{F l} \equiv \theta\left(\xi=0^{+}, y\right)$, which is found to be given by the expansion 


$$
\theta_{F l}=\left\{\begin{array}{l}
1-\frac{2 \gamma \Gamma}{\beta} y-\frac{l_{F}}{\beta U_{0}(\Gamma)} \varepsilon f_{0}^{\prime \prime}(y) \\
\text { at fuel-lean side } \\
1+\frac{2 \gamma(1-\Gamma)}{\beta} y-\frac{l_{O}}{\beta U_{0}(\Gamma)} \varepsilon f_{0}^{\prime \prime}(y) \\
\text { at fuel-rich side }
\end{array}\right.
$$

Significance of (47) is clear: The first term on the RHS, equal to 1 , is the flame temperature in the absence of gradients in the fresh mixture, that is, the adiabatic flame temperature of a planar stoichiometric flame. The second term, linear in $y$, describes the deviation of flame temperature for an infinitely thin flame $(\varepsilon=0)$, resulting from the linear deviation of mass fractions and of temperature in the fresh mixture from their values at the stoichiometric location (see the boundary conditions 5 ). The third term indicates the combined effects of flame curvature and differential diffusion. It is zero for unity Lewis numbers, positive for Lewis numbers less than 1 , and negative otherwise in agreement with available knowledge on stretched premixed flames (see Refs. [9] and [10], for example).

We close this section by specifying the location of the stoichiometric surface behind the premixed flame $\xi>0$ or position of the diffusion-flame tail of the triple flame. To this end, we determine the mass fractions behind the flame, $y_{F}^{b}$ and $y_{O}^{b}$, which are given by

$$
\begin{aligned}
y_{O}^{b}= & \frac{2 \gamma}{\beta} y+\frac{\left(l_{F}-l_{O}\right)}{\beta U_{0}(\Gamma)} \varepsilon f_{0}^{\prime \prime}(y) \\
& \text { at fuel-lean side } \\
y_{F}^{b}= & -\frac{2 \gamma}{\beta} y-\frac{\left(l_{F}-l_{O}\right)}{\beta U_{0}(\Gamma)} \varepsilon f_{0}^{\prime \prime}(y) \\
& \text { at fuel-rich side }
\end{aligned}
$$

At the location $y^{\text {st }}$ of the stoichiometric surface at $\xi$ $=0^{+}, y_{O}^{b}=y_{F}^{b}=0$. Hence,

$$
y^{s t}=0+\frac{\left(l_{O}-l_{F}\right)}{2 \gamma U_{0}(\Gamma)} \varepsilon f_{0}^{\prime \prime}(0)+\ldots
$$

Thus, the position of the stoichiometric surface behind the flame is shifted from the upstream stoichiometric surface, $y=0$, if the Lewis numbers of the fuel and oxidizer are different. Because $f^{\prime \prime}$ is positive, it is clear that the shift is toward the oxidizer side if $L e_{O}>L e_{F}$.

\section{Conclusion}

We have examined the propagation of a triple flame in a strained mixing layer formed between two streams of fuel and oxidizer, whose initial temperatures can differ. Allowing the Lewis numbers to deviate from unity, we have formulated the problem of triple-flame-front propagation for an overall Arrhenius reaction with large activation energy for the distinguished limiting case for which the ratio $\varepsilon$ of the thickness of the planar stoichiometric flame to the characteristic size of the flame-front region is of order unity. We may expect the propagation velocity $U$ to change from positive to negative and, therefore, the front from ignition front to extinction front of the diffusion flame, when $\varepsilon$ grows above a value $\varepsilon_{\mathrm{c}}$, of order $\sqrt{\beta}$, small compared with the characteristic value of extinction of the strained diffusion flame, of order $\beta$. For the cases $\varepsilon \ll 1$, analytical expressions determining the propagation speed of the triple flame, the local burning rate, and the flame shape have been obtained.

\section{Acknowledgments}

The present study was supported by the Commission of the European Communities within the frame of the Programme "Gravity Dependent Phenomena in Combustion," and postdoctoral Grant Number ERBFMBICT961503. We acknowledge also the support pf INTA under Contract INTA-UPM 'Mezcla y Combustíon'.

\section{REFERENCES}

1. Liñán, A., in Combustion in High Speed Flows (J. Buckmaster, T. L. Jackson, and A. Kumar, eds.), Kluwer Academic, Boston, 1994, p. 461.

2. Dold, J. W., Combust. Flame 76:71 (1989).

3. Hartley, L. J. and Dold J. W., Combust. Sci. Technol. 80:23 (1991).

4. Kioni, P. N., Rogg, B., Bray, C. and Liñán, A., Combust. Flame 95:276-290 (1993).

5. Ruetsch, G. R., Vervisch, L., and Liñán, A., Phys. Fluids 7 (6), (1995).

6. Buckmaster, J. and Matalon, M., in Twenty-Second Symposium (International) on Combustion, The Combustion Institute, Pittsburgh, 1988, pp. 1527-1535.

7. Matkowsky, B. J. and Sivashinsky, G. I., SIAM J. Appl. Math. 37:686 (1979).

8. Buckmaster, J. and Ludford, G. S., Lectures on Mathematical Combustion, SIAM Press, Philadelphia, 1983.

9. Clavin, P., Prog. Energy Combust. Sci. 11:1-59 (1985).

10. Matalon, M. and Matkowsky, B. J., J. Fluid. Mech. 124:239-259 (1982). 